\title{
Time Delay Estimation Method Used Median Filter in Low Signal to Noise Ratio Pulse Environment
}

\author{
Hang Liu ${ }^{1}$, Wenhong $\operatorname{Liu}^{2 *}$ \\ ${ }^{1}$ School of Electronic Information, Shanghai Dianji University, Shanghai, 200000, China \\ ${ }^{2}$ School of Electronic Information, Shanghai Dianji University, Shanghai, 200000, China
}

\begin{abstract}
In practice, the collected signal often contains impulsive noise. The classical time delay estimation algorithm based on the second-order statistics of Gaussian distribution will degrade or even be unreliable, so that it cannot be used. Although the fractional low-order signal processing method can be better adapted to signal processing in the impulse noise environment, the determination of the order $p$ value of the fractional low-order moment depends on the prior knowledge or estimation of the characteristic index $\alpha$ value of the pulse, and when the pulse is stronger or the signal-to-noise ratio is low, the performance cannot meet the requirements well. The paper adopted the method of median filter preprocessing. First, the abnormal points (pulse points) are removed in the noise and return the noise to the Gaussian model distribution; next, use the time delay estimation algorithm under the second-order statistics to avoid the estimate of p-value. Computer simulation experiments show that the method proposed in this paper has better estimation performance in low snr pulse environment.
\end{abstract}

\section{Introduction}

Since the furnace tube in the boiler is working in a high temperature, high pressure and corrosive environment for a long time, the furnace tube will often crack and cause leakage in large-scale coal-fired power plants. For the location of leaks, acoustic location [1] is used in practice; the time delay estimation location method based on the dual primitive model is also the most commonly used location method. The time difference (D) from the source to the sensor array, and D corresponds to the distance difference from the source to the sensor, to calculate the location of the leak. Therefore, the accuracy of time delay estimation plays a decisive role in the location of the final leak. Due to the complex working environment in the boiler, the noise is diverse, and different noise environments require different signal processing methods. This paper will focus on the time delay estimation method under the impulse noise environment [2].

Time Delay estimation algorithms are generally divided into four categories: correlation-based time delay estimation algorithms; phase-based delay estimation algorithms; time delay estimation algorithms based on Hibert transform; and adaptive estimation algorithms. Currently, since the basic correlation method is the most fully studied, simple and easy to implement, this algorithm is used in this paper. However, the time delay estimation of the correlation method is an algorithm based on secondorder statistics. When the signal noise obeys the normal distribution, the correlation method has better performance; when the noise signal is impulsive, it obviously not obeys the normal distribution, but fractional low-order $\alpha$ stable distribution noise. This moment, there is no limited second-order statistics in the pulse signal and the algorithm performance of the correlation method based on the second-order statistics will degrade or even not work normally [3]. It is necessary to study a new time delay estimation method with better toughness, when the degradation cannot be tolerated. Literature [4] proposed a series of time delay estimation methods based on fractional low-order statistics (FLOS), such as the time delay estimation algorithm based on covariance; the time delay estimation algorithm based on fractional low-order covariance; the least mean p-norm time delay estimation algorithm. For the fractional low-order moment (FLOM), it is defined as $E\left[|x|^{p}\right]$, where $0<\mathrm{p}<\alpha<=2$. Since the order $p$ of moments is often limited to the range of $(0, \alpha)$, the determination of the $p$ value depends on the understanding or estimation of the prior knowledge [5]; F LOS is dealing with the question of algebraic tailing, it does not provide a universal framework [4]. Therefore, although the aforementioned FLOS-based delay estimation method can adapt to the impulsiveness of noise, it is more difficult than the second-order statistics processing method when actually processing signals.

Based on this, the paper proposes a median filtering method in the signal preprocessing stage. First, median filtered is used to eliminate the noise in the pulse part of the entire signal [6] and adopt second-order Time delay estimation method of statistics. Computer simulation experiments show that the method can suppress impulse noise well and obtain more accurate time delay estimation.

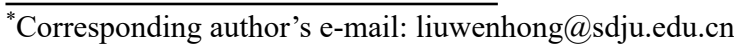




\section{Signal model}

\subsection{Median filter pretreatment}

Suppose the received signal is $x(n)$, which satisfies the following discrete signal model:

$$
\begin{gathered}
x_{1}(n)=s(n)+e_{1}(n) \\
x_{2}(n)=s(n-D)+e_{2}(n)
\end{gathered}
$$

Among them, $s(n)$ is a noise-free signal, $s(n-D)$ is a delayed signal relative to $s(n)$, and $D$ is the true value of the delay, $e_{1}(n), e_{2}(n)$ are impulse noise signal that obeys a stable distribution of $\alpha$, assuming that the three are statistically independent of each other.

Construct two received signals according to the signal and noise model. The noise-free signal $s(n)$ was generated by a 6th-order Butterworth low-pass filter with a bandwidth of 0.1 , and $s(n-D)$ was shifted to the right by the sequence of $s(n)$ by $D$ - One point was generated, and the impulse noise signal was simulated by the noise that obeys the stable distribution of $\alpha$ ( $\alpha$ is $1.7, \beta$ is $0, \gamma$ is 1 , and $\mu$ is 0 ), the initial delay value $D$ was set to 10 , and the length of the signal is 10,000 points. Figure 1 is the simulation sequence of $s(n)$; Figure 2 is the simulation sequence of $x_{1}(n)$, it can be seen that the signal has strong impulse.

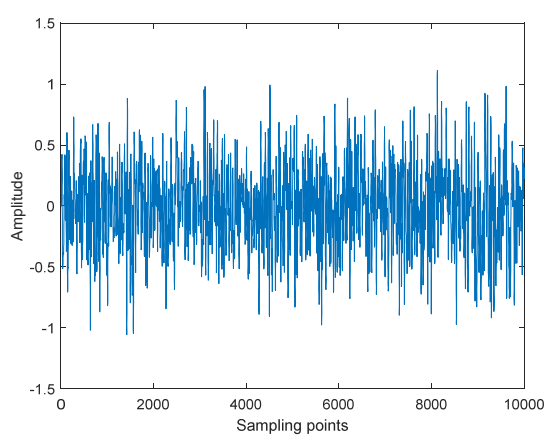

Figure 1.s(n)simulation sequence

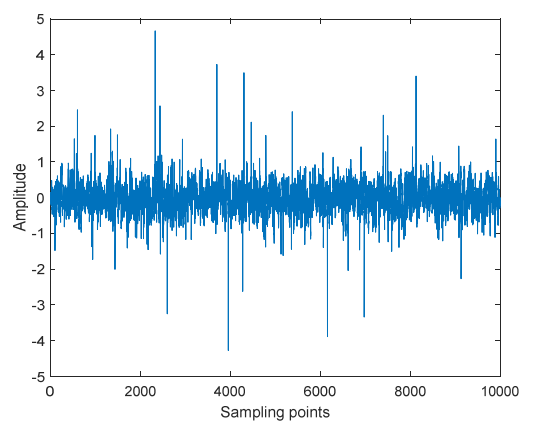

Figure 2. $x_{1}(n)$ simulation sequence

\subsection{Median Filter}

Impulse points are also called abnormal points in mathematics which are usually the maximum or minimum values in a set of data. By continuously extracting the median data, the abnormal points can be well filtered out[6]. Therefore, the median filter is a non-linear filter and has good suppression of impulse noise. In addition, the median filter algorithm is simple relatively, and hardware implementation is also easy relatively.

Perform median filtering on the one-dimensional signal $x_{n}(n=1,2, \ldots N)$. First, define a sliding window $\mathrm{M}$ ( $\mathrm{M}$ is an odd number), which slides on the domain. At a certain moment, the sample signals in the window are $x_{n-a}, \ldots, x_{n}, \ldots x_{n+a}$, where $x_{n}$ is the center position of the sample, and $a=(M-1) / 2$. The $\mathrm{M}$ sample signals are arranged in order from small to large or from large to small, and the number in the middle is selected as the filtered output. Which is:

$$
X_{n}=\operatorname{Mid}\left(x_{n-a}, \ldots, x_{n}, \ldots x_{n+a}\right)
$$

Therefore, it can be seen that the size of the window $M$ has a great impact on the final filtering effect; when the window size is too small, the noise reduction ability is limited; the window size is too large, the noise reduction ability can be improved, but it will destroy the details of the signal. In this regard, the method of multiple median filtering can be used to improve these defects. By performing multiple median filtering operations on all signal points in the filter window, the median value obtained is retained after each median filtering. Finally, perform the last median filtering operation on the retained median data, and the result obtained is the final result.

\section{Time Delay Estimation}

From Figure 4.2, it can be seen that the impulse signal is well suppressed after the median filter of the original impulse noise signal, and the noise signal returns to the normal distribution, and then the delay method based on second-order statistics is adopted. The method research is simple and easy to implement, so this article mainly adopts the correlation method and its improved algorithm to estimate the signal delay.

\subsection{Basic Correlation Method}

Calculate the correlation function between $x_{1}(n)$ and $x_{2}(n)$, there are:

$$
\begin{gathered}
R_{12}(\tau)=E\left[x_{1}(n) x_{2}(n+\tau)\right] \\
=R_{s s}(\tau-D)+R_{s e 1}(\tau-D) \\
+R_{s e 2}(\tau-D)+R_{e 1 e 2}(D)
\end{gathered}
$$

If the signal and noise satisfy the assumption that the three are uncorrelated, the three are completely orthogonal, then:

$$
\begin{aligned}
& R_{s e 1}(\tau-D)=0, \quad R_{s e 2}(\tau-D)=0, \quad R_{e 1 e 2}(D)=0 \\
& \text { inferred: } \\
& R_{12}(\tau)=R_{S S}(\tau-D)
\end{aligned}
$$

Known from the nature of the correlation function:

$$
\left|R_{S S}(\tau-D)\right| \leq R_{S S}(0)
$$

It is concluded that when $\tau=D$, the correlation function gets the maximum value. Therefore, the time delay estimation should choose $R_{S S}(\tau-D)$ to obtain the largest value of $\tau$ :

$$
\widehat{D}=\arg \left\{\max _{\tau}\left[R_{S S}(\tau-D)\right]\right\}
$$

Among them, $\arg \{$.$\} represents the argument of the$ function, and max [.] represents the maximum value of the function. 


\subsection{HB Weighted Correlation Adaptive Time Delay Method}

Although the median filter method can well suppress the impulse noise in the signal, the median filter does not have a good filtering effect for other types of noise. Therefore, the presence of noise will still cause the peak position to shift, or even false peaks in the time delay estimation. In this paper, the ratio of the signal peak to the background noise at the true value of the delay is the maximum criterion, and the $\mathrm{HB}$ weighting function is used as:

$$
H(f)=\frac{G_{s s}}{G_{x_{1} x_{1}}(f) G_{x_{2} x_{2}}(f)}
$$

HB-weighted generalized correlation method, the estimated variance can reach the lower bound of Cramero (CRLB). However, the optimal performance of this method depends on the prior knowledge of the noise signal and input statistics, which has certain difficulties in the actual process. Based on this, the article uses HB weighted adaptive time delay estimation method, referred to as LMSHB. The adaptive estimation method has the characteristics of automatically adjusting its structure and parameters and achieving the optimization under the control of the optimal criterion. Therefore, the adaptive method does not depend on the prior knowledge of the signal and noise, but it is easy Affected by noise. Therefore, LMSHB is a time delay estimation method that has good noise suppression capabilities and does not rely on statistical prior knowledge.

The HB weighted adaptive delay estimation method is based on the adaptive realization of two Roth processors. Under the assumption that the signal and noise are statistically independent of each other:

$$
\left|G_{x_{1} x_{2}}(f)\right|=G_{s s}(f)
$$

In this way, the $\mathrm{HB}$ weighting function can be written as:

$$
H(f)=\frac{\left|G_{x_{1} x_{2}}(f)\right|}{G_{x_{1} x_{1}}(f) G_{x_{2} x_{2}}(f)}
$$

Use the HB weighting function to weight the cross power spectral density function $G_{x_{1} x_{2}}(f)$ to obtain:

$$
\begin{gathered}
G_{y_{1} y_{2}}(f)=H(f) G_{x_{1} x_{2}}(f)=\frac{G_{x_{1} x_{2}}(f)\left|G_{x_{1} x_{2}}(f)\right|}{G_{x_{1} x_{1}}(f) G_{x_{2} x_{2}}(f)}(12) \\
\text { Since } H_{12}(f)=\frac{G_{x_{1} x_{2}}(f)}{G_{x_{1} x_{1}}(f)}, H_{21}(f)=\frac{G_{x_{2} x_{1}}(f)}{G_{x_{2} x_{2}}(f)}, \text { the above }
\end{gathered}
$$

formula can be rewritten as:

$$
G_{y_{1} y_{2}}(f)=\frac{G_{x_{1} x_{2}}(f)\left|G_{x_{1} x_{2}}(f)\right|}{G_{x_{1} x_{1}}(f) G_{x_{2} x_{2}}(f)}=H_{12}(f)\left|H_{21}(f)\right|
$$

In the formula, $H_{12}(f)$ and $H_{21}(f)$ are Roth processors, and the inverse Fourier transform is performed on the above formula to obtain the generalized correlation function:

$$
R_{y_{1} y_{2}}(\tau)=F^{-1}\left[G_{y_{1} y_{2}}(f)\right]=F^{-1}\left[H_{12}(f)\left|H_{21}(f)\right|\right](14)
$$

Among them, the adaptive realization of $H_{12}(f)$ and $H_{21}(f)$ can complete $\mathrm{HB}$ weighted adaptive delay estimation. In this way, the HB weighted generalized correlation method can be completely realized with an adaptive filter, namely:

$$
\hat{R}(\tau)=F^{-1}\left[W_{12}(f, n),\left|W_{21}(f, n)\right|\right]
$$

Among them, $W_{12}(f, n)$ and $W_{21}(f, n)$ represent the transfer functions of two adaptive filters, which simulate $H_{12}(f)$ and $H_{21}(f)$ respectively.

\subsection{Adaptive Least Mean p-norm Estimation Method (LMP)}

The LMP algorithm is evolved from the LMS (least mean square algorithm). The criterion for selecting the weight coefficients of the LMS is to minimize the mean square error $E\left[e^{2}(n)\right]$, the cost function used by the LMS is the square error; In impulsive environment, due to abnormal data such as extreme values in the sample, the square error is very sensitive to these abnormal data, which may cause the algorithm to fail to converge. The LMP method uses the p-norm of the error function to replace the squared error, the cost function of LMP is:

$$
J=E\left[|e(n)|^{p}\right]
$$

Suppose the output of the received signal vector $x_{1}(n)$ through the linear system $h(n)$ is $y(n)$, among them

$$
x_{1}(n)=
$$

$\left[x_{1}(n+L), x_{1}(n+L-1), \ldots, x_{1}(n), \ldots, x_{1}(n-L)\right]^{T}(17)$

$$
h(n)=[h(-L), h(-L+1), \ldots, h(0), \ldots, h(L)]^{T}
$$

The error function of the system is expressed as:

$$
\begin{gathered}
e(n)=x_{2}(n)-x_{1}^{T}(n) h(n) \\
=x_{2}(n)-\sum_{m=-L}^{L} h(m) x_{1}(n-m)
\end{gathered}
$$

Taking the p-norm of the error function $e(n)$ as the cost function:

$$
J=E\left(\left|x_{2}(n)-\sum_{m=-L}^{L} h(m) x_{1}(n-m)\right|^{p}\right)
$$

The theoretical formula of the cost function shown in formula (20) can be obtained by the estimation formula shown in formula (21)

$$
\hat{J}=\frac{1}{N-2 L} \sum_{n=L+1}^{N-L}\left|x_{2}(n)-\sum_{k=-L}^{L} h(m) x_{1}(n-m)\right|^{p}
$$

In the formula, $\mathrm{N}$ represents the sample size of the data. Using the steepest descent method to minimize the cost function, the adaptive iterative formula of the LMP algorithm can be obtained.

$$
h(n+1)=h(n)-\mu \widehat{\nabla}(n)
$$

Where $\widehat{\nabla}(n)$ is the estimate of the gradient $\nabla(n)$ at time $n$, expressed as:

$$
\begin{gathered}
\widehat{\nabla}(n)=\frac{\partial \hat{\jmath}}{\partial h(m)} \\
=-\frac{p \sum_{n=L+1}^{N-L} x_{1}(n)\left[x_{2}(n)-h^{T}(m) x_{1}(n)\right]^{<p-1>}}{N-2 L} \\
\text { The time delay is estimated as: } \\
\widehat{D}=-\underset{m}{\operatorname{argmin} \hat{j}[h(m)]}
\end{gathered}
$$

\section{Computer Simulation Experiment}

The following will use computer simulation experiments to compare the time delay estimation method using second-order statistics (LMSHB is mainly used here) and the estimation performance of the time delay estimation method that directly uses the fractional low-order statistics (the least mean p-norm (LMP) is mainly used here). 
Construct two received signals according to the signal and noise model. The noise-free signal $s(n)$ is generated by a 6th-order Butterworth low-pass filter with a bandwidth of 0.1 , and $s(n-D)$ is shifted to the right by the sequence of $s(n)$ by D- One point is generated, and the impulse noise signal is simulated by the noise that obeys a stable distribution of $\alpha$, and the initial delay value $D$ is set to 10 , and the length of the signal is 10000 points.

Experiment 1: Constantly adjusted the filter window size $\mathrm{M}$ of the median filter, compared the signal-to-noise ratio with the filter window size, and the relationship between the signal root mean square error and the filter window size, and selected the optimal filter window.
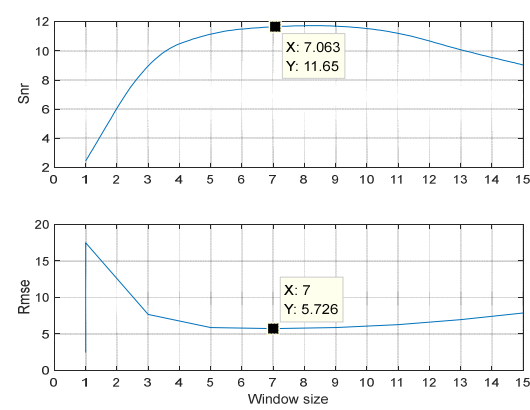

Figure 3. The influence of sliding window size on median filtering

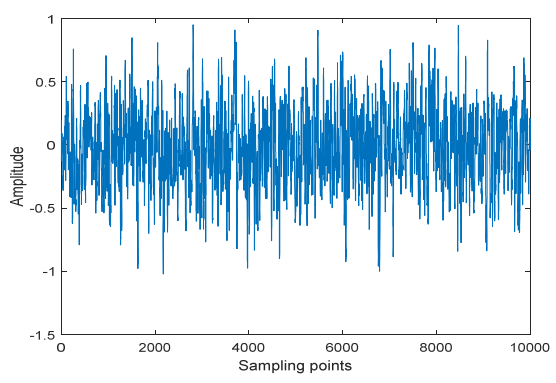

Figure 4. Filtered effect when the window size is 7

It can be seen from Figure 3 that as the filter window size increases, the signal-to-noise ratio of the filtered signal is also improving, indicating that the window size becomes larger, which can suppress the energy of noise, but when the size is too large, the data in the source signal is seriously lost, The signal-to-noise ratio will decrease accordingly; for the root mean square indicator, when the window size is 7 , the minimum root mean square error is 5.726. Therefore, in this paper, the sliding window of the median filter is set to 7 . Figure 4 shows the filtering effect when the window size is 7 . It can be seen that the median filter can suppress impulse noise better.

Experiment 2: Set the value of $\alpha$ to 1.8 (in a low impulse noise environment, the signal-to-noise ratio is $9.9455 \mathrm{db}$ ); the signal length was set to 10,000 points; the iteration step size set to 0.002 . Observe the estimated performance of these two methods.
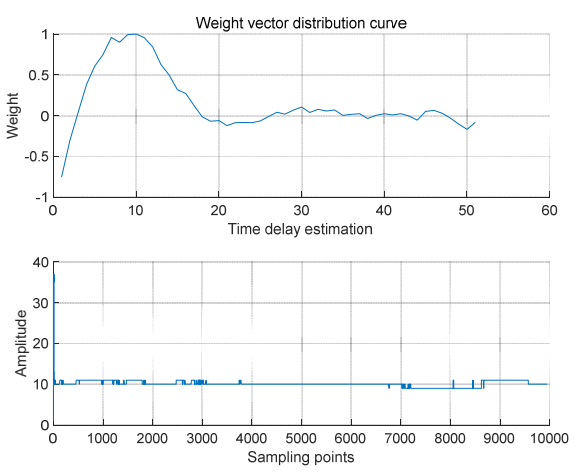

Figure $5 \alpha=1.8$, LMPTDE estimated performance
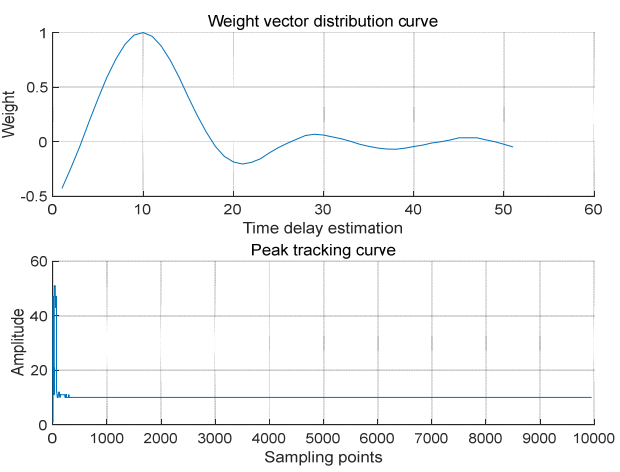

Figure 6. $\alpha=1.8$, LMSHB estimated performance

Table 1. Comparison of LMPTDE and LMSHB estimation performance $(\alpha=1.8)$

\begin{tabular}{lll}
\hline & $\begin{array}{l}\text { LMPTDE } \\
\text { Algorithm }\end{array}$ & $\begin{array}{l}\text { LMSHB } \\
\text { Algorithm }\end{array}$ \\
\hline $\begin{array}{l}\text { Estimated Mean } \\
\begin{array}{l}\text { Estimated } \\
\text { Variance }\end{array}\end{array}$ & 9.9312 & 10.0058 \\
\hline
\end{tabular}

From Figure 5, 6 and Table1, it can be seen that although the variance of the LMP method is large, the two methods have good estimates in terms of overall estimation performance in the low impulse noise environment.

Experiment 3: Set the value of $\alpha$ to 1.4 (in a high impulse noise environment, the signal-to-noise ratio is $8.5365 \mathrm{db}$ ); the signal length was set to 10,000 points; the iteration step size was set to 0.002 . When the noise impulse was stronger, observe the estimation performance of these two methods. 

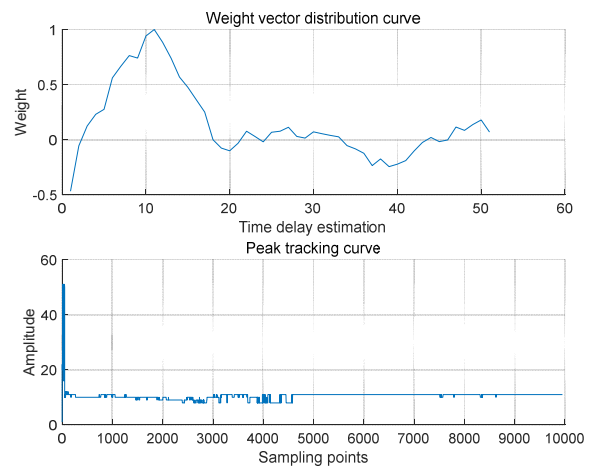

Figure 7. $\alpha=1.4$, LMPTDE estimated
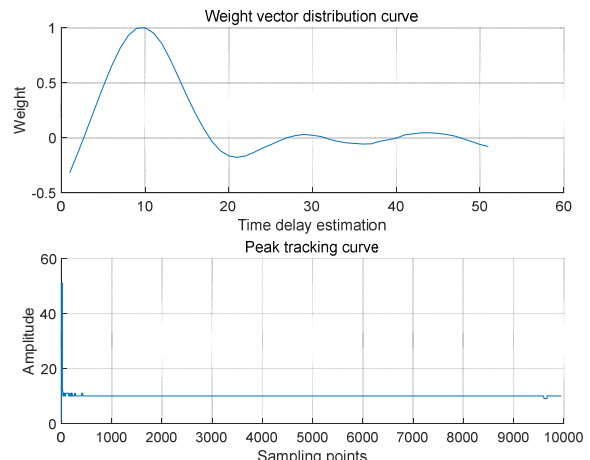

Figure 8. $\alpha=1.4$, LMSHB estimated performance

Table 2. Comparison of LMPTDE and LMSHB estimation performance $(\alpha=1.4)$

\begin{tabular}{lll}
\hline & $\begin{array}{l}\text { LMPTDE } \\
\text { Algorithm }\end{array}$ & $\begin{array}{l}\text { LMSHB } \\
\text { Algorithm }\end{array}$ \\
\hline $\begin{array}{l}\text { Estimated Mean } \\
\text { Estimated }\end{array}$ & 10.7097 & 10.0058 \\
Variance & 0.6707 & $3.3163 \mathrm{e}-05$ \\
\hline
\end{tabular}

From Figure 7, 8, and Table 2, it can be seen that when the impulsivity of the noise increases, the mean and variance estimated by the LMPTDE algorithm are significantly worse than when $\alpha=1.8$; As the impulsivity increases, the LMPTDE algorithm's estimation performance also declines. From the experimental results, it can be seen that it has good toughness no matter it is under low impulse noise conditions or under high impulse noise conditions after median filtering.

\section{Conclusion}

After the demonstration and analysis of this article, the median filter can be used to suppress the impulse noise. Then the signal processing method of the second-order statistics is used, the HB weighted generalized correlation method is used to suppress the noise, and the LMS adaptive method is combined. Experimental simulation shows that the second-order statistics processing method after median filtering has good toughness no matter in low impulse noise environment or high impulse noise environment.

\section{References}

1. R. N. Jing, (2020) Research on the location of four element sound source based on time delay estimation, Audio Engineering, vol. 44(3), pp. 21-23.

2. Y. B. Ding, (2019) Time delay estimation of cyclostationary signals under impulsive noise, Dalian: Dalian Maritime University, pp.1-75.

3. T. S. Qiu, (2020) Research progress of correlation entropy and cyclic correlation entropy signal processing, Journal of Electronics \& Information Technology, vol.42(1), pp.106-115

4. T. S. Qiu, X. X. Zhang and X. B. Li, Y. M. Sun, (2004) Statistical signal process-non gaussian signal processing and its application, Publishing House of Electronics Industry, Beijing.

5. Q. Yao, B. Wang, and L. J. Zhang, (2018) Ship identification analysis under alpha stable distribution noise," Journal of Signal Processing, vol.34(2), pp.175-181.

6. Z. X. Han, Y. H. Qiao, B. B. Li, and D. H. C, X. Wang, (2019) De-noising method of transmission line laser ranging signal based on median filtering and improved lifting wavelet," Power System \& Automation, vol.41(2), pp.45-48. 